\title{
Monotonicity-preserving rational bi-cubic spline surface interpolation
}

\author{
Muhammad Abbas ${ }^{\mathrm{a}, \mathrm{b}, *}$, Ahmad Abd Majid ${ }^{\mathrm{b}}$, Mohd Nain Hj Awang ${ }^{\mathrm{c}}$, Jamaludin Md Ali ${ }^{\mathrm{b}}$ \\ ${ }^{a}$ Department of Mathematics, University of Sargodha, 40100 Sargodha, Pakistan \\ b School of Mathematical Sciences, Universiti Sains Malaysia, 11800 USM Penang, Malaysia \\ c School of Distance Education, Universiti Sains Malaysia, 11800 USM Penang, Malaysia
}

*Corresponding author, e-mail: m.abbas@uos.edu.pk

Received 13 Feb 2014

Accepted 9 Jun 2014

\begin{abstract}
We discuss the problem of monotonicity preservation of surfaces through 3D monotone data. This can be done using a rational bi-cubic blended function that is an extension of a rational cubic function in the form of a cubic numerator and quadratic denominator. The function involves twelve shape parameters in each rectangular patch. Datadependent constraints are derived on four of these shape parameters to conserve the shape of the data while the other eight are left free to modify the monotone surface as desired. Several numerical examples are presented to show the effectiveness and capability of the scheme. The present scheme is $C^{1}$, flexible, simple, local, and economical.
\end{abstract}

KEYWORDS: rational bi-cubic blended function, monotone surface, 3D monotone data, shape parameters

\section{INTRODUCTION}

The problem of visualization of scientific data and shape preserving of curves or surfaces through known data points is one of the noteworthy problem. It has great significance in several fields like computer aided geometric design, computer graphics, engineering, medical, education, military, transport, advertising and meteorology. Some non-rational spline methods have developed to solve the aforementioned problem and to visualize the data well, but usually they pay no attention of innate shape features of data such as positivity, monotonicity and convexity. The objective of the present study is to conserve the monotonicity of surfaces through known scientific 3D monotone data. Monotone data arises in many physical situations from different sciences and art where entities only have a meaning when their values are monotone. For instance, erythrocyte sedimentation rates in cancer patients, approximation of couples and quasi couples in statistics, empirical option of pricing models in finance and dose-response curves and surfaces in biochemistry and pharmacology ${ }^{1}$.

Several researchers have focused on the problem of constructing shape preserving curves and surfaces through monotone data. A $C^{1}$ quadratic spline has been developed by Beatson and Ziegler in Ref. 2 to visualize the monotone data that was arranged over a rectangular grid. A bivariate interpolation has been extended to univariate interpolation developed by Carlson and Fritsch in Ref. 3 for monotone surface through regular data. The authors derived necessary and sufficient conditions on first partial derivatives and first mixed partial derivatives (twist) at knots using bi-cubic polynomial to conserve the monotonicity. Casciola and Romani ${ }^{4}$ preserved the shape of data by NURBS with tension parameters for rectangular case. Costantini and Fontanella ${ }^{5}$ developed interpolating splines of degree $n$ and order of continuity $k$ using Bernstein polynomials to construct the monotone and convex surfaces through arbitrary sets of data arranged on rectangular grid. Floater and Peña ${ }^{6}$ constructed three kinds of monotonicity-preserving of systems of bivariate functions with some geometric applications using Bernstein polynomials. Some researchers have developed rational cubic ${ }^{7,8}$ and rational bi-cubic ${ }^{9}$ spline interpolations to conserve the monotonicity through monotone data. Shape parameters have utilized in these interpolations to conserve and modify the shape of data as desired. Simple data dependent sufficient constraints were derived on shape parameters to conserve the monotonicity of data everywhere in the domain. Sarfraz ${ }^{10}$ visualized a monotone data in the view of monotone surfaces using the rational bi-cubic interpolant but it loses the smoothness of interpolant at some knots.

In this paper, a simple monotone $3 \mathrm{D}$ data visualization scheme is presented. We extend a $C^{1}$ piecewise rational cubic function with three shape parameters ${ }^{8}$ to rational bi-cubic blended function. It 
involves twelve shape parameters in each patch for its description. The degree of smoothness of proposed monotone surface interpolation is $C^{1}$. This paper adds to the knowledge domain by introducing a new cost effective, simple, local, easy to implement and time saving scheme which is more efficient for CAD systems than ever before as compared to existing schemes $^{9,10}$. As a result, the proposed rational bicubic blended function is simpler, easy to compute and implement and preserves the shape of surfaces with fewer constraints on shape parameters as compared to rational bi-cubic function ${ }^{9,10}$. The proposed surface scheme is unique in its representation and it works well for both equally and unequally space data.

\section{REVIEW OF RATIONAL CUBIC FUNCTION}

In this section, a piecewise rational function ${ }^{8}$ with three shape parameters can be rewritten in the form of (cubic/quadratic) to conserve the monotonicity of curves. Moreover, it is used to extend as rational bicubic blended function to conserve the monotonicity of surfaces through 3D monotone data.

Let $\left\{\left(t_{i}, f_{i}\right), i=0,1,2, \ldots, n\right\}$ be the given set of data points such as $t_{0}<t_{1}<t_{2}<\ldots<t_{n}$. A piecewise rational cubic function with three shape parameters, in each subinterval $\left[t_{i}, t_{i+1}\right], i=0,1,2, \ldots, n-1$ can be defined as

$$
P_{i}(t)=\frac{\sum_{k=0}^{3}(1-\varphi)^{3-k} \varphi^{k} \lambda_{k}}{r_{i}(\varphi)}
$$

where $r_{i}(\varphi)=\alpha_{i}(1-\varphi)^{2}+\left(\alpha_{i}+\beta_{i}+\gamma_{i}\right) \varphi(1-\varphi)+$ $\gamma_{i} \varphi^{2}$ and $\varphi=\left(t-t_{i}\right) / h_{i}, h_{i}=t_{i+1}-t_{i}$. Let $P_{i}^{\prime}(t)$ denote the first order derivative with respect to $t$. The following conditions are imposed on a function (1) for $C^{1}$ continuity

$$
\begin{aligned}
& P_{i}\left(t_{i}\right)=f_{i}, \quad P_{i}\left(t_{i+1}\right)=f_{i+1} \\
& P_{i}^{\prime}\left(t_{i}\right)=d_{i}, \quad P_{i}^{\prime}\left(t_{i+1}\right)=d_{i+1} .
\end{aligned}
$$

The unknown coefficients $\lambda_{k}, k=0,1,2,3$ can be calculated from equation (2),

$$
\begin{aligned}
& \lambda_{0}=\alpha_{i} f_{i}, \\
& \lambda_{1}=f_{i}\left(2 \alpha_{i}+\beta_{i}+\gamma_{i}\right)+\alpha_{i} h_{i} d_{i}, \\
& \lambda_{2}=f_{i+1}\left(\alpha_{i}+\beta_{i}+2 \gamma_{i}\right)-\gamma_{i} h_{i} d_{i+1}, \\
& \lambda_{3}=\gamma_{i} f_{i+1}
\end{aligned}
$$

where $\alpha_{i}, \beta_{i}$ and $\gamma_{i}$ are positive shape parameters and $d_{i}$ denote the derivative values that are used for smoothness of required curve. A piecewise rational cubic function (1) becomes a standard cubic Hermite spline when we set the values of shape parameters as $\alpha_{i}=1, \beta_{i}=0$ and $\gamma_{i}=1$. The following result was proposed in Ref. 8 .
Theorem 1 (Ref. 8) A piecewise rational cubic function (1) conserves the monotonicity of curves through monotone data, if in each subinterval $\left[t_{i}, t_{i+1}\right], i=$ $0,1,2, \ldots, n-1$, the shape parameters satisfy the following conditions

$$
\begin{aligned}
& \alpha_{i}>0, \gamma_{i}>0 \\
& \beta_{i}>\max \left\{0, \frac{\gamma_{i} d_{i+1}-\Delta_{i}\left(\alpha_{i}+2 \gamma_{i}\right)}{\Delta_{i}},\right. \\
& \left.\frac{\alpha_{i} d_{i}-\Delta_{i}\left(2 \alpha_{i}+\gamma_{i}\right)}{\Delta_{i}}\right\},
\end{aligned}
$$

where $\Delta_{i}=\left(f_{i+1}-f_{i}\right) / h_{i}$. The above result can be rearranged as

$$
\begin{aligned}
& \alpha_{i}>0, \gamma_{i}>0, \\
& \beta_{i}=r_{i}+\max \left\{0, \frac{\gamma_{i} d_{i+1}-\Delta_{i}\left(\alpha_{i}+2 \gamma_{i}\right)}{\Delta_{i}},\right. \\
& \left.\frac{\alpha_{i} d_{i}-\Delta_{i}\left(2 \alpha_{i}+\gamma_{i}\right)}{\Delta_{i}}\right\}
\end{aligned}
$$

for some $r_{i}>0$.

Remark 1 A piecewise rational cubic function (1) preserves a $C^{1}$ monotone curve through monotone data if the derivative values $d_{i}, i=0,1,2, \ldots, n$ are calculated at each knots by using arithmetic mean method proposed in Ref. 7.

Remark 2 The $C^{2}$ monotone curve through monotone data can be obtained by using piecewise rational cubic function (1) with derivative values $d_{i}, i=$ $0,1,2, \ldots, n$ when they are calculated at each knots by solving the system of linear equations given in Ref. 8 .

Example 1 A monotone data set is taken in Table 1 for the test of monotonicity-preserving curves. Fig. 1 is generated by cubic Hermite spline scheme ${ }^{11}$ that does not conserve the monotonicity of monotone data. Fig. 2 is generated by using Piecewise Cubic Hermite Interpolating Polynomial (PCHIP, Built-in MATLAB program) to conserve the monotonicity of same data but the visual model is not smooth at some data points which may not be suitable for practical design. On the other hand, Fig. 3 and Fig. 4 are generated

Table 1 Monotone data set.

\begin{tabular}{cccccccccccc}
\hline$i$ & 1 & 2 & 3 & 4 & 5 & 6 & 7 & 8 & 9 & 10 & 11 \\
\hline$t_{i}$ & 0.1 & 4 & 6.5 & 10 & 15 & 25 & 40 & 50 & 62 & 65 & 66 \\
$f_{i}$ & 1 & 1 & 2 & 3.5 & 5.5 & 5.5 & 10 & 10 & 12.5 & 18 & 20 \\
\hline
\end{tabular}




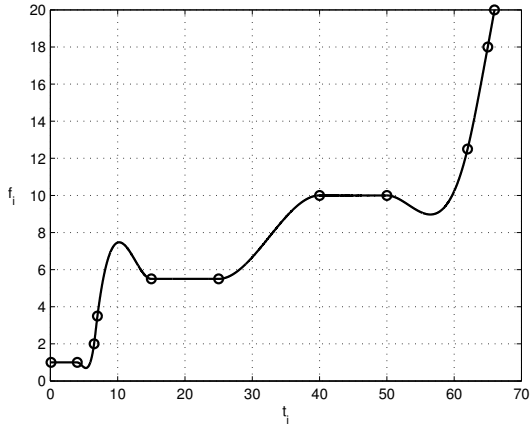

Fig. 1 Cubic Hermite spline curve.

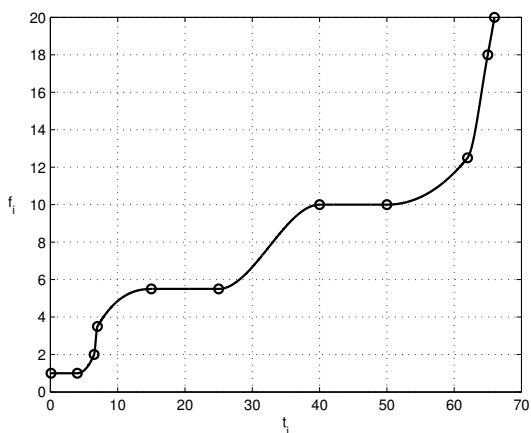

Fig. 2 PCHIP curve.

by rational cubic function (1) with shape parameters and derivative values calculated from Theorem 1 and arithmetic mean method ${ }^{7}$, respectively, to conserve the monotonicity. The effect of shape parameters can be seen by noting the difference in $C^{1}$ smoothness of these monotonicity-preserving curves in Fig. 2, Fig. 3, and Fig. 4.

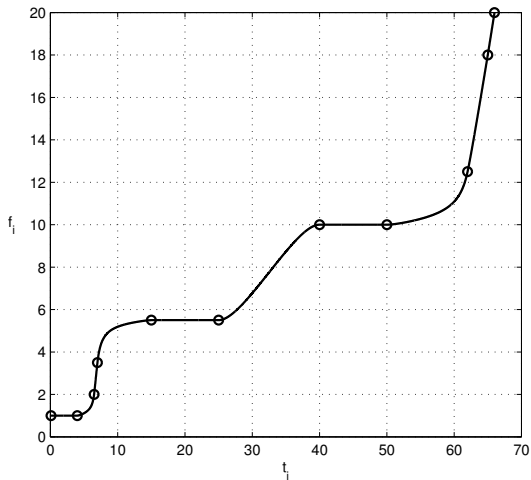

Fig. 3 A $C^{1}$ monotone curve using rational cubic function with $\alpha_{i}=\beta_{i}=0.05$ and $r_{i}=0.1$.

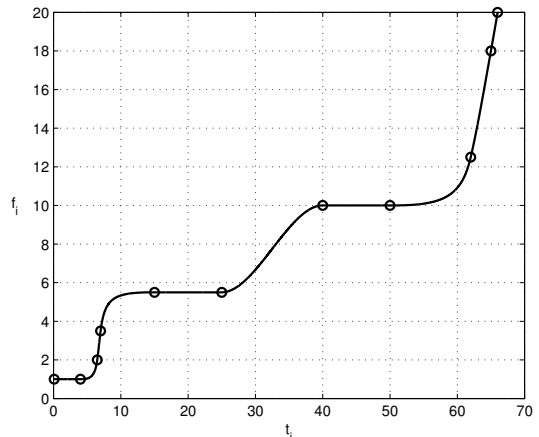

Fig. 4 A $C^{1}$ monotone curve using rational cubic function with $\alpha_{i}=\beta_{i}=0.5$ and $r_{i}=0.1$.

\section{RATIONAL BI-CUBIC BLENDED SPLINE FUNCTION}

The problem of constructing a rectangular coons surface patches from given network of boundary curves is discussed in this section. This job is done using rational bi-cubic blended function which is an extension of rational cubic function (1). The rational bi-cubic blended function is suitable to arrange the data over rectangular mesh $\left[\xi, \xi_{1}\right] \times\left[\sigma, \sigma_{1}\right]$ such that $\xi=t_{0}<t_{1}<\ldots<t_{n}=\xi_{1}$ and $\sigma=u_{0}<u_{1}<\ldots<$ $u_{m}=\sigma_{1}$. The rational bi-cubic blended function over each patch $\left[t_{i}, t_{i+1}\right] \times\left[u_{j}, u_{j+1}\right], i=0,1,2, \ldots, n-1$, $j=0,1,2, \ldots, m-1$ is defined as

$$
\begin{aligned}
& P(t, u)= {\left[m_{0}(\varphi), m_{1}(\varphi)\right]\left[\begin{array}{c}
P\left(t_{i}, u\right) \\
P\left(t_{i+1}, u\right)
\end{array}\right] } \\
&+\left[n_{0}(\phi), n_{1}(\phi)\right]\left[\begin{array}{c}
P\left(t, u_{j}\right) \\
P\left(t, u_{j+1}\right)
\end{array}\right] \\
&-\left[m_{0}(\varphi), m_{1}(\varphi)\right]\left[\begin{array}{cc}
F_{i, j} & F_{i, j+1} \\
F_{i+1, j} & F_{i+1, j+1}
\end{array}\right]\left[\begin{array}{l}
n_{0}(\phi) \\
n_{1}(\phi)
\end{array}\right]
\end{aligned}
$$

with

$$
\begin{aligned}
m_{0}(\varphi) & =(1-\varphi)^{2}(1+2 \varphi), m_{1}(\varphi)=\varphi^{2}(3-2 \varphi), \\
n_{0}(\phi) & =(1-\phi)^{2}(1+2 \phi), n_{1}(\phi)=\phi^{2}(3-2 \phi),
\end{aligned}
$$

are called Hermite blending functions with $\varphi=(t-$ $\left.t_{i}\right) / h_{i}, \phi=\left(u-u_{j}\right) / \hat{h}_{j}$, where $h_{i}=t_{i+1}-t_{i}, \hat{h}_{j}=$ $u_{j+1}-u_{j}$ and $P\left(t, u_{j}\right), P\left(t, u_{j+1}\right), P\left(t_{i}, u\right)$, and $P\left(t_{i+1}, u\right)$ are four boundary rational cubic curves of rectangular patch.

The boundary rational cubic curves can be defined as

$$
P\left(t, u_{j}\right)=\frac{\sum_{i=0}^{3}(1-\varphi)^{3-i} \varphi^{i} \delta_{i}}{r_{1}(\varphi)}
$$


with

$$
\begin{aligned}
\delta_{0}= & \alpha_{i, j} F_{i, j}, \\
\delta_{1}= & \left(2 \alpha_{i, j}+\beta_{i, j}+\gamma_{i, j}\right) F_{i, j}+\alpha_{i, j} h_{i} F_{i, j}^{t}, \\
\delta_{2}= & \left(\alpha_{i, j}+\beta_{i, j}+2 \gamma_{i, j}\right) F_{i+1, j}-\gamma_{i, j} h_{i} F_{i+1, j}^{t}, \\
\delta_{3}= & \gamma_{i, j} F_{i+1, j}, \\
r_{1}(\varphi)= & \alpha_{i, j}(1-\varphi)^{2} \\
& +\left(\alpha_{i, j}+\beta_{i, j}+\gamma_{i, j}\right) \varphi(1-\varphi)+\gamma_{i, j} \varphi^{2} ; \\
& P\left(t, u_{j+1}\right)=\frac{\sum_{i=0}^{3}(1-\varphi)^{3-i} \varphi^{i} \eta_{i}}{r_{2}(\varphi)}
\end{aligned}
$$

with

$$
\begin{aligned}
\eta_{0}= & \alpha_{i, j+1} F_{i, j+1}, \\
\eta_{1}= & \left(2 \alpha_{i, j+1}+\beta_{i, j+1}+\gamma_{i, j+1}\right) F_{i, j+1} \\
& +\alpha_{i, j+1} h_{i} F_{i, j+1}^{t}, \\
\eta_{2}= & \left(\alpha_{i, j+1}+\beta_{i, j+1}+2 \gamma_{i, j+1}\right) F_{i+1, j+1} \\
& -\gamma_{i, j+1} h_{i} F_{i+1, j+1}^{t}, \\
\eta_{3}= & \gamma_{i, j+1} F_{i+1, j+1}, \\
r_{2}(\varphi)= & \alpha_{i, j+1}(1-\varphi)^{2}+\gamma_{i, j+1} \varphi^{2} \\
& +\left(\alpha_{i, j+1}+\beta_{i, j+1}+\gamma_{i, j+1}\right) \varphi(1-\varphi) ; \\
& P\left(t_{i}, u\right)=\frac{\sum_{i=0}^{3}(1-\phi)^{3-i} \phi^{i} \mu_{i}}{r_{3}(\phi)}
\end{aligned}
$$

with

$$
\begin{aligned}
\mu_{0}= & \hat{\alpha}_{i, j} F_{i, j}, \\
\mu_{1}= & \left(2 \hat{\alpha}_{i, j}+\hat{\beta}_{i, j}+\hat{\gamma}_{i, j}\right) F_{i, j}+\hat{\alpha}_{i, j} \hat{h}_{j} F_{i, j}^{u}, \\
\mu_{2}= & \left(\hat{\alpha}_{i, j}+\hat{\beta}_{i, j}+2 \hat{\gamma}_{i, j}\right) F_{i, j+1}-\hat{\gamma}_{i, j} \hat{h}_{j} F_{i, j+1}^{u}, \\
\mu_{3}= & \hat{\gamma}_{i, j} F_{i, j+1}, \\
r_{3}(\phi)= & \hat{\alpha}_{i, j}(1-\phi)^{2} \\
& +\left(\hat{\alpha}_{i, j}+\hat{\beta}_{i, j}+\hat{\gamma}_{i, j}\right) \phi(1-\phi)+\hat{\gamma}_{i, j} \phi^{2} ; \\
& P\left(t_{i+1}, u\right)=\frac{\sum_{i=0}^{3}(1-\phi)^{3-i} \phi^{i} \psi_{i}}{r_{4}(\phi)}
\end{aligned}
$$

with

$$
\begin{aligned}
\psi_{0}= & \hat{\alpha}_{i, j+1} F_{i+1, j}, \\
\psi_{1}= & \left(2 \hat{\alpha}_{i, j+1}+\hat{\beta}_{i, j+1}+\hat{\gamma}_{i, j+1}\right) F_{i+1, j} \\
& +\hat{\alpha}_{i, j+1} \hat{h}_{j} F_{i+1, j}^{u}, \\
\psi_{2}= & \left(\hat{\alpha}_{i, j+1}+\hat{\beta}_{i, j+1}+2 \hat{\gamma}_{i, j+1}\right) F_{i+1, j+1} \\
& -\hat{\gamma}_{i, j+1} \hat{h}_{j} F_{i+1, j+1}^{u}, \\
\psi_{3}= & \hat{\gamma}_{i+1, j} F_{i+1, j+1}, \\
r_{4}(\phi)= & \hat{\alpha}_{i, j+1}(1-\phi)^{2}+\hat{\gamma}_{i, j+1} \phi^{2} \\
& +\left(\hat{\alpha}_{i, j+1}+\hat{\beta}_{i, j+1}+\hat{\gamma}_{i, j+1}\right) \phi(1-\phi) .
\end{aligned}
$$

\section{MONOTONICITY-PRESERVING RATIONAL BI-CUBIC BLENDED SPLINE INTERPOLATION}

In this section, we discuss the monotonicitypreserving surface problem. We use rational bicubic blended function (3) with shape parameters and derived simple data dependent constraints on these shape parameters to assure the monotonicity of surfaces through 3D monotone data. Let $\left\{\left(t_{i}, u_{j}, F_{i, j}\right), i=0,1,2, \ldots, n ; j=0,1,2, \ldots, m\right\}$ be given set of monotone data is arranged over rectangular mesh $\left[t_{i}, t_{i+1}\right] \times\left[u_{j}, u_{j+1}\right], i=0,1,2, \ldots, n-1$, $j=0,1,2, \ldots, m-1$, such that $F_{i, j}<F_{i+1, j}, F_{i, j}<$ $F_{i, j+1}$ or equivalently $\Delta_{i, j}>0, \hat{\Delta}_{i, j}>0$ and $F_{i, j}^{t}>$ $0, F_{i, j}^{u}>0$ for all $i, j$. The necessary conditions on shape parameters for monotonicity are

$$
\begin{array}{r}
\alpha_{i, j}, \alpha_{i, j+1}, \hat{\alpha}_{i, j}, \hat{\alpha}_{i+1, j}>0 \\
\gamma_{i, j}, \gamma_{i, j+1}, \hat{\gamma}_{i, j}, \hat{\gamma}_{i+1, j}>0 .
\end{array}
$$

According to the result developed in Ref. 4, the rational bi-cubic blended surface patch inherits all the properties of network of boundary curves. Hence the rational bi-cubic blended function (3) conserves the monotonicity of surfaces through $3 \mathrm{D}$ monotone data if the four boundary curves $P\left(t, u_{j}\right), P\left(t, u_{j+1}\right)$, $P\left(t_{i}, u\right)$, and $P\left(t_{i+1}, u\right)$ defined in equations (4)(7) are monotone. The boundary curve $P\left(t, u_{j}\right)$ is monotone if $P^{\prime}\left(t, u_{j}\right)>0$, i.e.,

$$
P^{\prime}\left(t, u_{j}\right)=\frac{\sum_{i=0}^{4}(1-\varphi)^{4-i} \varphi^{i} \chi_{i}}{\left(r_{1}(\varphi)\right)^{2}}>0
$$

with

$$
\begin{aligned}
& \chi_{0}= \alpha_{i, j}^{2} F_{i, j}^{t}, \\
& \chi_{1}= 2 \alpha_{i, j}\left[\left(\alpha_{i, j}+\beta_{i, j}+2 \gamma_{i, j}\right) \Delta_{i, j}-\gamma_{i, j} F_{i+1, j}^{t}\right], \\
& \chi_{2}= \chi_{1}+\chi_{3}+\left(\chi_{0}+\chi_{4}\right) \\
&+\beta_{i, j}\left(\alpha_{i, j}+\beta_{i, j}+\gamma_{i, j}\right) \Delta_{i, j} \\
&-2 \alpha_{i, j} \gamma_{i, j}\left(F_{i, j}^{t}+F_{i+1, j}^{t}\right), \\
& \chi_{3}= 2 \gamma_{i, j}\left[\left(2 \alpha_{i, j}+\beta_{i, j}+\gamma_{i, j}\right) \Delta_{i, j}-\alpha_{i, j} F_{i, j}^{t}\right], \\
& \chi_{4}= \gamma_{i, j}^{2} F_{i+1, j}^{t}, \\
& \text { and } \sum_{i=0}^{4}(1-\varphi)^{4-i} \varphi^{i} \chi_{i}>0 \text { if } \chi_{i}>0 \text { for } i= \\
& 0,1,2,3,4 . \operatorname{Thus} \chi_{i}>0 \text { if } \\
& \beta_{i, j}>\max \left\{0, \frac{\gamma_{i, j} F_{i+1, j}^{t}-\Delta_{i, j}\left(\alpha_{i, j}+2 \gamma_{i, j}\right)}{\Delta_{i, j}},\right. \\
&\left.\quad \frac{\alpha_{i, j} F_{i, j}^{t}-\Delta_{i, j}\left(2 \alpha_{i, j}+\gamma_{i, j}\right)}{\Delta_{i, j}}\right\} .
\end{aligned}
$$


Similarly, the boundary curve $P\left(t, u_{j+1}\right)$ is monotone, if $P^{\prime}\left(t, u_{j+1}\right)>0$, i.e.,

$$
P^{\prime}\left(t, u_{j+1}\right)=\frac{\sum_{i=0}^{4}(1-\varphi)^{4-i} \varphi^{i} \kappa_{i}}{\left(r_{2}(\varphi)\right)^{2}}>0
$$

with

$$
\begin{aligned}
\kappa_{0}= & \alpha_{i, j+1}^{2} F_{i, j+1}^{t}, \\
\kappa_{1}= & 2 \alpha_{i, j+1}\left[\left(\alpha_{i, j+1}+\beta_{i, j+1}+2 \gamma_{i, j+1}\right] \Delta_{i, j+1}\right. \\
& \left.-\gamma_{i, j+1} F_{i+1, j+1}^{t}\right), \\
\kappa_{2}= & \kappa_{1}+\kappa_{3}+\left(\kappa_{0}+\kappa_{4}\right) \\
& +\beta_{i, j+1}\left(\alpha_{i, j+1}+\beta_{i, j+1}+\gamma_{i, j+1}\right) \Delta_{i, j+1} \\
& -2 \alpha_{i, j+1} \gamma_{i, j+1}\left(F_{i, j+1}^{t}+F_{i+1, j+1}^{t}\right), \\
\kappa_{3}= & 2 \gamma_{i, j+1}\left[\left(2 \alpha_{i, j+1}+\beta_{i, j+1}+\gamma_{i, j+1}\right) \Delta_{i, j+1}\right. \\
& \left.-\alpha_{i, j+1} F_{i, j+1}^{t}\right], \\
\kappa_{4}= & \gamma_{i, j+1}^{2} F_{i+1, j+1}^{t},
\end{aligned}
$$

and $\sum_{i=0}^{4}(1-\varphi)^{4-i} \varphi^{i} \kappa_{i}>0$, if $\kappa_{i}>0$ for $i=$ $0,1,2,3,4$. Thus $\kappa_{i}>0$ if

$$
\begin{aligned}
& \beta_{i, j+1}>\max \{0, \\
& \frac{\gamma_{i, j+1} F_{i+1, j+1}^{t}-\Delta_{i, j+1}\left(\alpha_{i, j+1}+2 \gamma_{i, j+1}\right)}{\Delta_{i, j+1}}, \\
& \left.\frac{\alpha_{i, j+1} F_{i, j+1}^{t}-\Delta_{i, j+1}\left(2 \alpha_{i, j+1}+\gamma_{i, j+1}\right)}{\Delta_{i, j+1}}\right\} .
\end{aligned}
$$

Similarly, the boundary curve $P\left(t_{i}, u\right)$ is monotone, if $P^{\prime}\left(t_{i}, u\right)>0$, i.e.,

$$
P^{\prime}\left(t_{i}, u\right)=\frac{\sum_{i=0}^{4}(1-\phi)^{4-i} \phi^{i} \lambda_{i}}{\left(r_{3}(\phi)\right)^{2}}>0
$$

with

$$
\begin{aligned}
\lambda_{0}= & \hat{\alpha}_{i, j}^{2} F_{i, j}^{u} \\
\lambda_{1}= & 2 \hat{\alpha}_{i, j}\left(\left(\hat{\alpha}_{i, j}+\hat{\beta}_{i, j}+2 \hat{\gamma}_{i, j}\right) \hat{\Delta}_{i, j}-\hat{\gamma}_{i, j} F_{i, j+1}^{u}\right), \\
\lambda_{2}= & \lambda_{1}+\lambda_{3}+\left(\lambda_{0}+\lambda_{4}\right) \\
& +\hat{\beta}_{i, j}\left(\hat{\alpha}_{i, j}+\hat{\beta}_{i, j}+\hat{\gamma}_{i, j}\right) \hat{\Delta}_{i, j} \\
& -2 \hat{\alpha}_{i, j} \hat{\gamma}_{i, j}\left(F_{i, j}^{u}+F_{i, j+1}^{u}\right) \\
\lambda_{3}= & 2 \hat{\gamma}_{i, j}\left(\left(2 \hat{\alpha}_{i, j}+\hat{\beta}_{i, j}+\hat{\gamma}_{i, j}\right) \hat{\Delta}_{i, j}-\hat{\alpha}_{i, j} F_{i, j}^{u}\right), \\
\lambda_{4}= & \hat{\gamma}_{i, j}^{2} F_{i, j+1}^{u}
\end{aligned}
$$

and $\sum_{i=0}^{4}(1-\phi)^{4-i} \phi^{i} \lambda_{i}>0$, if $\lambda_{i}>0$ for $i=$ $0,1,2,3,4$. Thus $\lambda_{i}>0$, if

$$
\begin{aligned}
\hat{\beta}_{i, j}>\max \left\{0, \frac{\hat{\gamma}_{i, j} F_{i, j+1}^{u}-\hat{\Delta}_{i, j}\left(\hat{\alpha}_{i, j}+2 \hat{\gamma}_{i, j}\right)}{\hat{\Delta}_{i, j}},\right. \\
\left.\frac{\hat{\alpha}_{i, j} F_{i, j}^{u}-\hat{\Delta}_{i, j}\left(2 \hat{\alpha}_{i, j}+\hat{\gamma}_{i, j}\right)}{\hat{\Delta}_{i, j}}\right\} .
\end{aligned}
$$

Finally, the boundary curve $P\left(t_{i+1}, u\right)$ is monotone, if $P^{\prime}\left(t_{i+1}, u\right)>0$, i.e.,

$$
P^{\prime}\left(t_{i+1}, u\right)=\frac{\sum_{i=0}^{4}(1-\phi)^{4-i} \phi^{i} \mu_{i}}{\left(r_{4}(\mu)\right)^{2}}>0
$$

with

$$
\begin{aligned}
\mu_{0}= & \hat{\alpha}_{i+1, j}^{2} F_{i+1, j}^{u}, \\
\mu_{1}= & 2 \hat{\alpha}_{i+1, j}\left[\left(\hat{\alpha}_{i+1, j}+\hat{\beta}_{i+1, j}+2 \hat{\gamma}_{i+1, j}\right) \hat{\Delta}_{i+1, j}\right. \\
& \left.-\hat{\gamma}_{i+1, j} F_{i+1, j+1}^{u}\right] \\
\mu_{2}= & \mu_{1}+\mu_{3}+\left(\mu_{0}+\mu_{4}\right) \\
& +\hat{\beta}_{i+1, j}\left(\hat{\alpha}_{i+1, j}+\hat{\beta}_{i+1, j}+\hat{\gamma}_{i+1, j}\right) \hat{\Delta}_{i+1, j} \\
& -2 \hat{\alpha}_{i+1, j} \hat{\gamma}_{i+1, j}\left(F_{i+1, j}^{u}+F_{i+1, j+1}^{u}\right), \\
\mu_{3}= & 2 \hat{\gamma}_{i+1, j}\left[\left(2 \hat{\alpha}_{i+1, j}+\hat{\beta}_{i+1, j}+\hat{\gamma}_{i+1, j}\right) \hat{\Delta}_{i+1, j}\right. \\
& \left.-\hat{\alpha}_{i+1, j} F_{i+1, j}^{u}\right] \\
\mu_{4}= & \hat{\gamma}_{i+1, j}^{2} F_{i+1, j+1}^{u},
\end{aligned}
$$

and $\sum_{i=0}^{4}(1-\phi)^{4-i} \phi^{i} \mu_{i}>0$, if $\mu_{i}>0$ for $i=$ $0,1,2,3,4$. Thus $\mu_{i}>0$, if

$$
\begin{aligned}
& \hat{\beta}_{i+1, j}>\max \{0, \\
& \frac{\hat{\gamma}_{i+1, j} F_{i+1, j+1}^{u}-\hat{\Delta}_{i+1, j}\left(\hat{\alpha}_{i+1, j}+2 \hat{\gamma}_{i+1, j}\right)}{\hat{\Delta}_{i+1, j}}, \\
&\left.\frac{\hat{\alpha}_{i+1, j} F_{i+1, j}^{u}-\hat{\Delta}_{i+1, j}\left(2 \hat{\alpha}_{i+1, j}+\hat{\gamma}_{i+1, j}\right)}{\hat{\Delta}_{i+1, j}}\right\}
\end{aligned}
$$

where

$\Delta_{i, j}=\left(F_{i+1, j}-F_{i, j}\right) / h_{i}, \hat{\Delta}_{i, j}=\left(F_{i, j+1}-F_{i, j}\right) / \hat{h}_{j}$.

Theorem 2 The rational bi-cubic blended function (3) conserves the monotonicity of surfaces through $3 D$ monotone data, if in each rectangular patch $\left[t_{i}, t_{i+1}\right] \times\left[u_{j}, u_{j+1}\right], \quad i=0,1,2, \ldots, n-1, \quad j=$ $0,1,2, \ldots, m-1$ the shape parameters are satisfying the following sufficient conditions,

$$
\begin{array}{r}
\beta_{i, j}>\max \left\{0, \frac{\gamma_{i, j} F_{i+1, j}^{t}-\Delta_{i, j}\left(\alpha_{i, j}+2 \gamma_{i, j}\right)}{\Delta_{i, j}},\right. \\
\left.\frac{\alpha_{i, j} F_{i, j}^{t}-\Delta_{i, j}\left(2 \alpha_{i, j}+\gamma_{i, j}\right)}{\Delta_{i, j}}\right\},
\end{array}
$$




$$
\begin{array}{r}
\beta_{i, j+1}>\max \{0, \\
\frac{\gamma_{i, j+1} F_{i+1, j+1}^{t}-\Delta_{i, j+1}\left(\alpha_{i, j+1}+2 \gamma_{i, j+1}\right)}{\Delta_{i, j+1}}, \\
\left.\frac{\alpha_{i, j+1} F_{i, j+1}^{t}-\Delta_{i, j+1}\left(2 \alpha_{i, j+1}+\gamma_{i, j+1}\right)}{\Delta_{i, j+1}}\right\}, \\
\hat{\beta}_{i, j}>\max \left\{0, \frac{\hat{\gamma}_{i, j} F_{i, j+1}^{u}-\hat{\Delta}_{i, j}\left(\hat{\alpha}_{i, j}+2 \hat{\gamma}_{i, j}\right)}{\hat{\Delta}_{i, j}},\right. \\
\left.\frac{\hat{\alpha}_{i, j} F_{i, j}^{u}-\hat{\Delta}_{i, j}\left(2 \hat{\alpha}_{i, j}+\hat{\gamma}_{i, j}\right)}{\hat{\Delta}_{i, j}}\right\}
\end{array}
$$

and

$$
\begin{aligned}
& \hat{\beta}_{i+1, j}>\max \{0, \\
& \frac{\hat{\gamma}_{i+1, j} F_{i+1, j+1}^{u}-\hat{\Delta}_{i+1, j}\left(\hat{\alpha}_{i+1, j}+2 \hat{\gamma}_{i+1, j}\right)}{\hat{\Delta}_{i+1, j}}, \\
& \left.\frac{\hat{\alpha}_{i+1, j} F_{i+1, j}^{u}-\hat{\Delta}_{i+1, j}\left(2 \hat{\alpha}_{i+1, j}+\hat{\gamma}_{i+1, j}\right)}{\hat{\Delta}_{i+1, j}}\right\} .
\end{aligned}
$$

The above results can be expressed as

$$
\begin{gathered}
\beta_{i, j}=r_{i, j}+\max \{0, \\
\frac{\gamma_{i, j} F_{i+1, j}^{t}-\Delta_{i, j}\left(\alpha_{i, j}+2 \gamma_{i, j}\right)}{\Delta_{i, j}}, \\
\left.\frac{\alpha_{i, j} F_{i, j}^{t}-\Delta_{i, j}\left(2 \alpha_{i, j}+\gamma_{i, j}\right)}{\Delta_{i, j}}\right\}, \\
\beta_{i, j+1}=s_{i, j}+\max \{0, \\
\frac{\gamma_{i, j+1} F_{i+1, j+1}^{t}-\Delta_{i, j+1}\left(\alpha_{i, j+1}+2 \gamma_{i, j+1}\right)}{\Delta_{i, j+1}}, \\
\left.\frac{\alpha_{i, j+1} F_{i, j+1}^{t}-\Delta_{i, j+1}\left(2 \alpha_{i, j+1}+\gamma_{i, j+1}\right)}{\Delta_{i, j+1}}\right\}, \\
\hat{\beta}_{i+1, j}=\hat{s}_{i, j}+\max \{0, \\
\left.\frac{\hat{\alpha}_{i, j} F_{i, j}^{u}-\hat{\Delta}_{i, j}\left(2 \hat{\alpha}_{i, j}+\hat{\gamma}_{i, j}\right)}{\hat{\Delta}_{i, j}}\right\} \\
\hat{r}_{i, j}+\max \left\{\begin{array}{l}
0, \hat{\Delta}_{i, j}\left(\hat{\alpha}_{i, j}+2 \hat{\gamma}_{i, j}\right) \\
\hat{\beta}_{i, j}
\end{array}\right\}
\end{gathered}
$$

$$
\begin{gathered}
\frac{\hat{\gamma}_{i+1, j} F_{i+1, j+1}^{u}-\hat{\Delta}_{i+1, j}\left(\hat{\alpha}_{i+1, j}+2 \hat{\gamma}_{i+1, j}\right)}{\hat{\Delta}_{i+1, j}}, \\
\left.\frac{\hat{\alpha}_{i+1, j} F_{i+1, j}^{u}-\hat{\Delta}_{i+1, j}\left(2 \hat{\alpha}_{i+1, j}+\hat{\gamma}_{i+1, j}\right)}{\hat{\Delta}_{i+1, j}}\right\},
\end{gathered}
$$

for some $r_{i, j}, s_{i, j}, \hat{r}_{i, j}, \hat{s}_{i, j}>0$.

Proof: The result follows immediately from the above discussion.

\section{Algorithm 1}

(i) Given a monotone data set $\left\{\left(t_{i}, u_{j}, F_{i, j}\right), i=\right.$ $0,1,2, \ldots, n ; j=0,1,2, \ldots, m\}$.

(ii) Calculate the partial derivatives $F_{i, j}^{t}, F_{i, j}^{u}$, for $i=0,1,2, \ldots, n, j=0,1,2, \ldots, m$ at given data points using arithmetic mean method for 3D data proposed in Ref. 9.

(iii) Choose any positive value for free shape parameters $\alpha_{i, j}, \alpha_{i, j+1}, \hat{\alpha}_{i, j}, \hat{\alpha}_{i+1, j}, \gamma_{i, j}, \gamma_{i, j+1}, \hat{\gamma}_{i, j}$, $\hat{\gamma}_{i+1, j}$ and then calculate the values of constrained shape parameters $\beta_{i, j}, \beta_{i, j+1}, \hat{\beta}_{i, j}, \hat{\beta}_{i+1, j}$ using Theorem 2.

(iv) Compute the rational boundaries $P\left(t, u_{j}\right)$, $P\left(t, u_{j+1}\right), P\left(t_{i}, u\right)$, and $P\left(t_{i+1}, u\right)$ defined in equations (4)-(7) for the rectangular patches.

(v) Insert the calculated values of (i), (ii), (iii), and (iv) in the rational bi-cubic blended function (3) to obtain the monotonicity-preserving surfaces.

\section{NUMERICAL EXAMPLES}

In this section, a rational bi-cubic blended function (3) with shape parameters is employed to conserve the monotonicity preserving of surfaces through given monotone data. Several numerical examples are presented in this section to exhibit the capability and effectiveness of the proposed monotone rational bicubic blended interpolation. A desired smooth and visually pleasing monotone surface obtained from several examples is compared with bi-cubic Hermite interpolant ${ }^{11}$.

Example 2 The data set taken in Table 2 is produced from the following function

$$
F(t, u)=\exp \left(t^{0.05}+u^{0.05}\right)
$$

Table 2 3D monotone data generated from function (17).

\begin{tabular}{lccccc}
\hline$t / u$ & 0 & 2 & 6 & 10 & 14 \\
\hline 0 & 1 & 2.82 & 2.98 & 3.07 & 3.13 \\
2 & 2.82 & 7.93 & 8.41 & 8.65 & 8.81 \\
6 & 2.98 & 8.41 & 8.91 & 9.17 & 9.34 \\
10 & 3.07 & 8.65 & 9.17 & 9.43 & 9.61 \\
14 & 3.13 & 8.81 & 9.34 & 9.61 & 9.79 \\
\hline
\end{tabular}




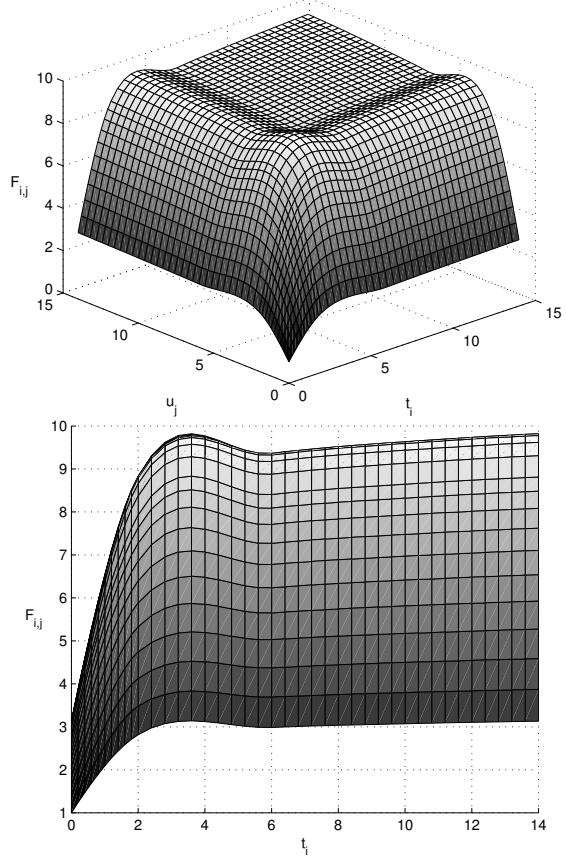

Fig. 5 Bi-cubic Hermite spline surface, and $x z$-view.

Fig. 5 is produced using bi-cubic Hermite spline ${ }^{11}$ that does not conserve the monotonicity. To remove this flaw, Fig. 6 depicts the monotone surface everywhere in the domain using developed rational bi-cubic blended interpolant with shape parameters $\alpha_{i, j}=\hat{\alpha}_{i, j}=\gamma_{i, j}=\hat{\gamma}_{i, j}=0.5$.

Example 3 The 3D monotone data set which is taken in Table 3 for the test of monotonicity-preserving of surfaces. Fig. 7 displays non-monotone surface through given monotone data using bi-cubic Hermite spline ${ }^{11}$. This flaw can be removed well in Fig. 8 when it is produced by using developed monotone rational bi-cubic blended scheme with shape parameters $\alpha_{i, j}=\hat{\alpha}_{i, j}=\gamma_{i, j}=\hat{\gamma}_{i, j}=0.5$.

Example 4 A 3D monotone data set in Table 4 is taken from following mathematical function,

$$
F(t, u)=t^{2}\left(t^{10}+1\right)+u^{2}\left(u^{10}+1\right)
$$

Table 3 A monotone data set.

\begin{tabular}{lccccc}
\hline$t / u$ & 1 & 2 & 3 & 4 & 5 \\
\hline 1 & 0.2098 & 0.2099 & 0.2100 & 0.2101 & 0.2102 \\
2 & 0.9437 & 0.9438 & 0.9439 & 0.9440 & 0.9441 \\
3 & 0.9986 & 0.9987 & 0.9988 & 0.9989 & 0.9990 \\
4 & 0.9994 & 0.9995 & 0.9996 & 0.9997 & 0.9998 \\
5 & 1.0001 & 1.0002 & 1.0003 & 1.0004 & 1.0005 \\
\hline
\end{tabular}

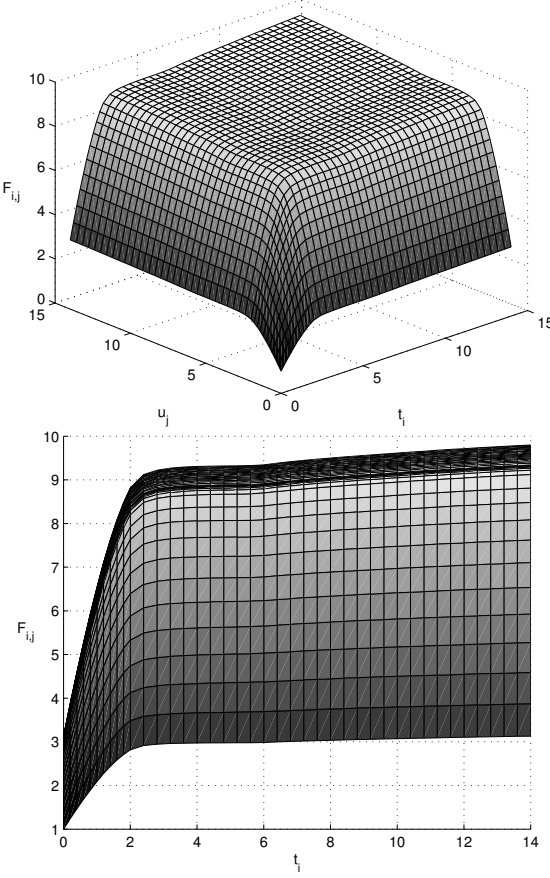

Fig. 6 A monotone surface by rational bi-cubic blended spline function with $r_{i, j}=\hat{r}_{i, j}=s_{i, j}=\hat{s}_{i, j}=0.01$, and $x z$-view.

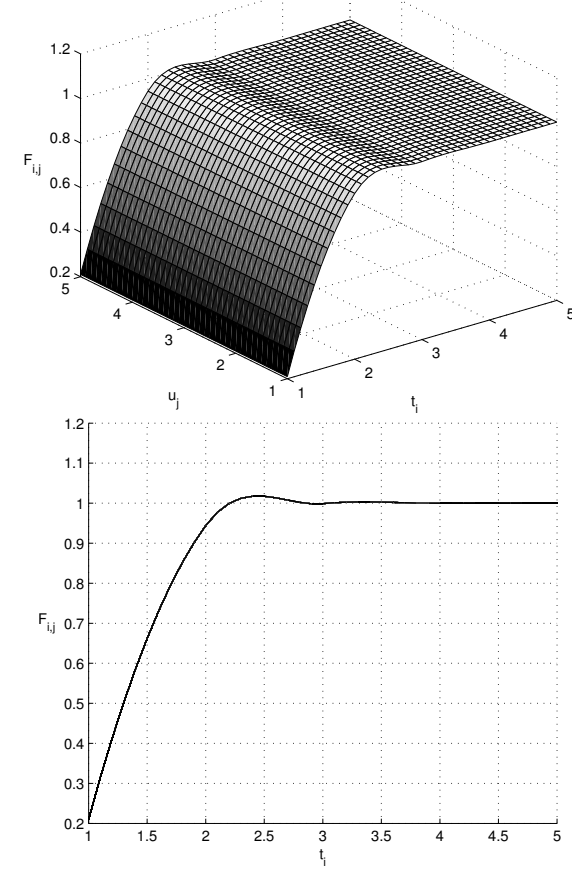

Fig. 7 Surface generated by bi-cubic Hermite spline, and $x z$-view. 


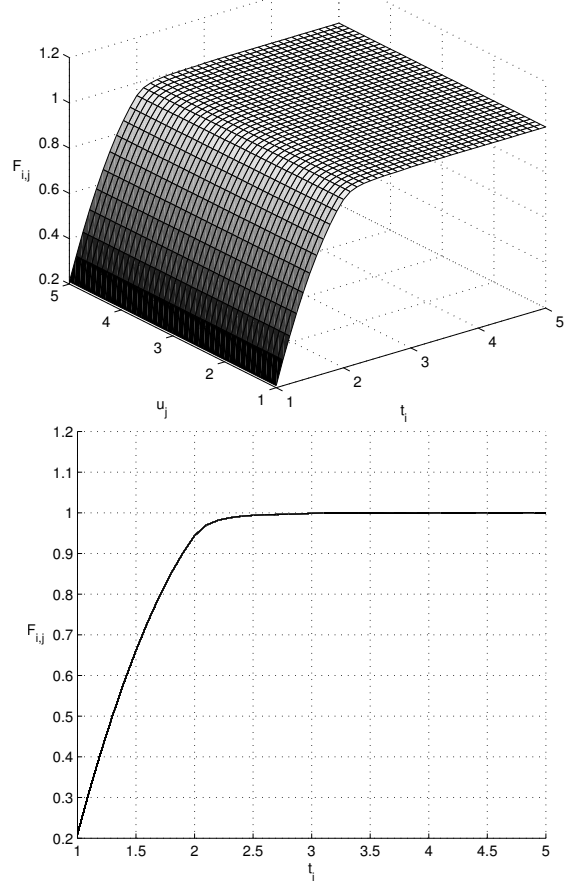

Fig. 8 Monotone surface developed by rational bi-cubic blended spline function with $r_{i, j}=\hat{r}_{i, j}=s_{i, j}=\hat{s}_{i, j}=0.1$, and $x z$-view.

Table 4 A 3D monotone data set.

\begin{tabular}{cccccc}
\hline$t / u$ & 0.2 & 0.5 & 0.9 & 1.5 & 2.0 \\
\hline 0.2 & 0.08 & 0.29 & 1.13 & 132.04 & 4100.0 \\
0.5 & 0.29 & 0.50 & 1.34 & 132.25 & 4100.3 \\
0.9 & 1.13 & 1.34 & 2.18 & 133.09 & 4101.1 \\
1.5 & 132.04 & 132.25 & 133.09 & 263.99 & 4232.0 \\
2.0 & 4100.0 & 4100.3 & 4101.1 & 4232.0 & 8200.0 \\
\hline
\end{tabular}

Fig. 9 can be drawn using bi-cubic Hermite spline ${ }^{11}$. It depicts non-monotone surface at some knots. On other hand, Fig. 10 can be produced using developed monotone rational bi-cubic blended scheme. It preserves the monotonicity everywhere in the domain with values of shape parameters $\alpha_{i, j}=\hat{\alpha}_{i, j}=\gamma_{i, j}=$ $\hat{\gamma}_{i, j}=0.05$.

\section{CONCLUDING REMARKS}

In this paper, we have extended a $C^{1}$ piecewise rational cubic function ${ }^{8}$ to rational bi-cubic blended function to conserve the shape of 3D monotone data in the view of monotone surface. Twelve shape parameters are used in each rectangular patch and they are arranged in such a way; four of them are constrained parameters while the remaining eight are

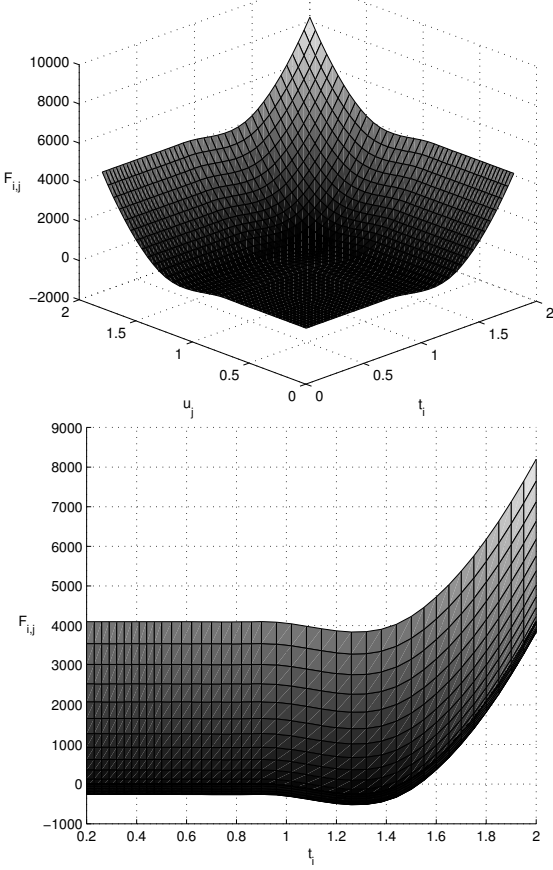

Fig. 9 Non monotone surface using bi-cubic Hermite spline, and $x z$-view.
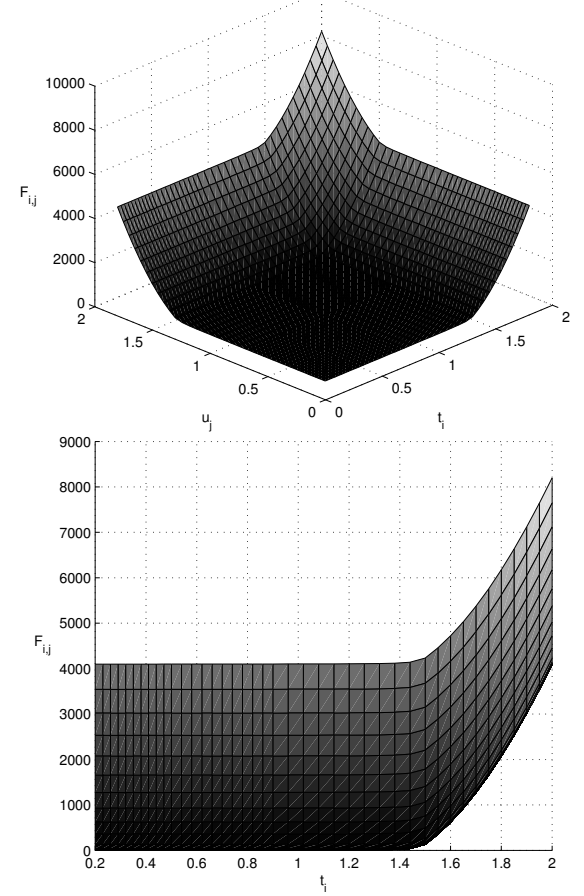

Fig. 10 Monotonicity-preserving surface using rational bicubic blended spline function with $r_{i, j}=\hat{r}_{i, j}=s_{i, j}=$ $\hat{s}_{i, j}=0.001$, and $x z$-view. 
left free for designer's choice for the modification of monotone surface as desired. The proposed scheme is suitable for such problems in which only data points are given; in contrast the schemes ${ }^{5,6}$ imposed a set of constraints on derivatives and data points to obtain the required shape of data. The developed surface scheme has been demonstrated through different numerical examples and observed that the scheme is not only local and computationally economical but is also visually pleasant compared to existing schemes ${ }^{9,10}$.

Acknowledgements: The authors are grateful to the anonymous reviewers for their helpful, valuable comments and suggestions to improve this paper. This work was fully supported by FRGS Grant No. 203/PMATHS/6711324 from the School of Mathematical Sciences, Universiti Sains Malaysia.

\section{REFERENCES}

1. Beliakov G (2005) Monotonicity preserving approximation of multivariate scattered data. BIT 45, 653-77.

2. Beatson RK, Ziegler Z (1985) Monotonicity preserving surface interpolation. SIAM J Numer Anal 22, 401-11.

3. Carlson RE, Fritsch FN (1985) Monotone piecewise bicubic interpolation. SIAM J Numer Anal 22, 386-400.

4. Casciola G, Romani L (2003) Rational interpolants with tension parameters. In: Lyche T, Mazure ML, Schumaker LL (eds) Proceedings of Curve and Surface Design, Saint-Malo, Nashboro Press, Brentwood, TN, pp 41-50.

5. Costantini P, Fontanella F (1990) Shape-preserving bivariate interpolation. SIAM J Numer Anal 27, 488-506.

6. Floater MS, Peña JM (2000) Monotonicity preservation on triangles. Math Comput 69, 1505-19.

7. Abbas M, Majid AA, Awang MNH, Ali JM (2011) Monotonicity preserving interpolation using rational spline. In: Ao SI, Castillo O, Douglas C, Feng DD, Lee JA (eds) Proceedings of the International MultiConference of Engineers and Computer Scientists 2011, pp 278-82.

8. Abbas M, Majid AA, Ali JM (2012) Monotonicitypreserving $C^{2}$ rational cubic spline for monotone data. Appl Math Comput 219, 2885-95.

9. Abbas M, Majid AA, Awang MNH, Ali JM (2012) Shape-preserving rational bi-cubic spline for monotone surface data. WSEAS Trans Math 7, 660-73.

10. Sarfraz M, Butt S, Hussain MZ (1997) Surfaces for the visualization of scientific data preserving monotonicity. In: Goodman T, Martin R (eds) Proceedings of the IMA Mathematics for Surfaces VII. pp 479-95.

11. Hoschek J, Lasser D (1993) Fundamentals of Computer Aided Geometric Design, translated by Schumaker LL. A K Peters, Wellesley, MA. 